\title{
The Medial Prefrontal Cortex Is Involved in Spatial Memory Retrieval under Partial-Cue Conditions
}

\author{
Yong Sang Jo, ${ }^{1}$ Eun Hye Park, ${ }^{1}$ Il Hwan Kim, ${ }^{2}$ Soon Kwon Park, ${ }^{3}$ Hyun Kim, ${ }^{2}$ Hyun Taek Kim, ${ }^{1}$ and June-Seek Choi ${ }^{1}$ \\ ${ }^{1}$ Department of Psychology, Korea University, Seoul 136-701, Korea, ${ }^{2}$ Department of Anatomy, Korea University College of Medicine, Seoul, Korea, and \\ ${ }^{3}$ School of Alternative Medicine and Health Science, Jeonju University, Jeonju 520-759, Korea
}

\begin{abstract}
Brain circuits involved in pattern completion, or retrieval of memory from fragmented cues, were investigated. Using different versions of the Morris water maze, we explored the roles of the CA3 subregion of the hippocampus and the medial prefrontal cortex (mPFC) in spatial memory retrieval under various conditions. In a hidden platform task, both CA3 and mPFC lesions disrupted memory retrieval under partial-cue, but not under full-cue, conditions. For a delayed matching-to-place task, CA3 lesions produced a deficit in both forming and recalling spatial working memory regardless of extramaze cue conditions. In contrast, damage to mPFC impaired memory retrieval only when a fraction of cues was available. To corroborate the lesion study, we examined the expression of the immediate early gene c-fos in mPFC and the hippocampus. After training of spatial reference memory in full-cue conditions for $6 \mathrm{~d}$, the same training procedure in the absence of all cues except one increased the number of Fos-immunoreactive cells in mPFC and CA3. Furthermore, mPFC inactivation with muscimol, a GABA agonist, blocked memory retrieval in the degraded-cue environment. However, mPFC-lesioned animals initially trained in a single-cue environment had no difficulty in retrieving spatial memory when the number of cues was increased, demonstrating that contextual change per se did not impair the behavioral performance of the mPFC-lesioned animals. Together, these findings strongly suggest that pattern completion requires interactions between $\mathrm{mPFC}$ and the hippocampus, in which $\mathrm{mPFC}$ plays significant roles in retrieving spatial information maintained in the hippocampus for efficient navigation.
\end{abstract}

Key words: prefrontal cortex; CA3; spatial memory; pattern completion; memory retrieval; water maze; c-fos

\section{Introduction}

Only rarely does memory retrieval occur in the presence of a complete set of cues used during memory formation. Most of the time, degraded cues are provided and yet they are sufficient to activate the entire memory. Theoretical models suggest that the ability to recall an entire memory from its fragments can be achieved through associative recall or pattern completion in the CA3 area of the hippocampus, which forms an associative memory network with extensive recurrent collaterals (Marr, 1971; Treves and Rolls, 1994; Hasselmo et al., 1995). In support of this idea, lesion and NMDA receptor knock-out in CA3 disrupt animals' performance in finding target locations under partial-cue conditions (Nakazawa et al., 2002; Gold and Kesner, 2005), and CA3 neurons display coherent activity response between the original and cue-altered environment (Lee et al., 2004; Vazdarjanova and Guzowski, 2004). Thus, CA3 plays an essential role in spatial pattern completion.

Few studies have investigated the possibility that brain structures other than CA3 might be involved in pattern completion.

Received Aug. 7, 2007; revised 0ct. 11, 2007; accepted 0ct. 26, 2007.

This work was supported by the Korea Ministry of Science and Technology under Korea Science and Engineering Foundation Grants M10644020003-06N4402-00310 and R01-2004-000-10613-0 (J.-S.C.) and by 21st Century Frontier Research Program Grant M103KV010025-07K2201-02510.

Correspondence should be addressed to June-Seek Choi, Department of Psychology, Korea University, Anamdong, Seongbuk-gu, Seoul 136-701, Korea. E-mail: j-schoi@korea.ac.kr.

DOI:10.1523/JNEUROSCI.3589-07.2007

Copyright $\odot 2007$ Society for Neuroscience ～0270-6474/07/2713567-12\$15.00/0
Considering its dynamic interactions with the hippocampus, the medial prefrontal cortex (mPFC) is another candidate structure. This idea has been developed by observations that the neural activity of $\mathrm{mPFC}$ is highly correlated with activity in hippocampal cells (Jones and Wilson, 2005; Siapas et al., 2005) and that the hippocampus and $\mathrm{mPFC}$ compensate each other for processing of spatial memory (Lee and Kesner, 2003). Moreover, it has been observed that neurons in the prelimbic/infralimbic area (PL/IL) of mPFC display goal- or location-specific firing properties similar to hippocampal place cells (O'Keefe and Dostrovsky, 1971; Hok et al., 2005). In addition, experimental evidence regarding extra-hippocampal structures for reorganization of hippocampus-dependent memory indicates that the anterior cingulate cortex $(\mathrm{Cgl})$ and PL/IL of MPFC are crucial for the consolidation and expression of remote memory, respectively (Frankland et al., 2004; Maviel et al., 2004).

The mPFC consists of several interconnected regions. Among them, PL/IL receives the highest density of direct projections from CA1 and the subiculum (Jay and Witter, 1991; Conde et al., 1995). Moreover, long-term potentiation and depression exist in the hippocampo-prefrontal pathway, and such altered synaptic plasticity correlates with behavioral performance (Burette et al., 1997; Farinelli et al., 2006). Thus, mPFC (PL/IL area) as one of the main output structures of the hippocampus is expected to be implicated in spatial pattern completion. To address this, we trained rats with $\mathrm{mPFC}$ or CA3 lesions in two different types of the Morris water maze task with a complete set of cues (Morris et 
al., 1982) and then tested their retrieval performance in the incomplete-cue environment. Using c-fos immunohistochemistry, we also examined whether partial-cue removal activates neurons in mPFC. Finally, the GABA receptor agonist muscimol was infused into $\mathrm{mPFC}$ to examine its role, specifically in the expression of spatial memory under the partial-cue condition. These experiments allowed us to determine that $\mathrm{mPFC}$ may be required for spatial memory retrieval when available cues were reduced.

\section{Materials and Methods}

Subjects. Male Sprague Dawley rats (260-320 g; Orient Bio, Kyunggi-do, Korea) were housed individually in standard plastic cages. They were maintained on a $12 \mathrm{~h} \mathrm{light/dark} \mathrm{cycle} \mathrm{with} \mathrm{access} \mathrm{to} \mathrm{food} \mathrm{and} \mathrm{water} \mathrm{ad}$ libitum. All behavioral procedures were conducted during the light phase of the cycle.

Surgery. Animals were deeply anesthetized with sodium pentobarbital $(60 \mathrm{mg} / \mathrm{kg}$, i.p.) and placed on a stereotaxic instrument (David Kopf Instruments, Tujunga, CA). The scalp of each rat was incised, and the skull was adjusted to place bregma and lambda on the same horizontal plane. Small burr holes were drilled for either electrolytic lesions or the implantation of cannulas. For electrolytic lesions, stainless-steel electrodes $(0.3 \mathrm{~mm}$ in diameter) insulated with Epoxylite, except for $0.7 \mathrm{~mm}$ at the tip, were lowered bilaterally into the brain according to the following coordinates: (1) dorsal CA3 lesions: (a) $2.5 \mathrm{~mm}$ posterior, $2.6 \mathrm{~mm}$ lateral, and $3.4 \mathrm{~mm}$ ventral to bregma; (b) $3.3 \mathrm{~mm}$ posterior, $3.3 \mathrm{~mm}$ lateral, and $3.8 \mathrm{~mm}$ ventral to bregma, (c) $4.1 \mathrm{~mm}$ posterior, 4.0 mm lateral, and $4.2 \mathrm{~mm}$ ventral to bregma; (2) mPFC lesions: (a) $2.5 \mathrm{~mm}$ anterior, $0.6 \mathrm{~mm}$ lateral, and $4.0 \mathrm{~mm}$ ventral to bregma, (b) $3.5 \mathrm{~mm}$ posterior, $0.6 \mathrm{~mm}$ lateral, and $3.8 \mathrm{~mm}$ ventral to bregma (Paxinos and Watson, 1998). When the electrodes were in place, $1 \mathrm{~mA}, 15 \mathrm{~s}$ current was passed through the electrode. Sham groups received the same surgical procedures with exception that no current was passed. As for the implantation of cannulas, 26 gauge double guide cannulas (Plastics One, Roanoke, VA) were placed in $\mathrm{mPFC}: 3.0 \mathrm{~mm}$ anterior, $0.6 \mathrm{~mm}$ lateral, and $2.8 \mathrm{~mm}$ ventral to bregma (Paxinos and Watson, 1998). The cannulas were secured in place with anchoring screws and dental cement. Thirty-three gauge dummy cannulas were inserted into the $0.5 \mathrm{~mm}$ ventral of the guide cannulas to prevent clogging. The scalp was then closed with suture. The animals were allowed to recover for at least $7 \mathrm{~d}$, during which they were handled daily.

Water maze apparatus. Behavioral testing was conducted in a circular metal water maze tank $(157 \mathrm{~cm}$ in diameter, $60 \mathrm{~cm}$ in height) that was filled with water $\left(24 \pm 1^{\circ} \mathrm{C}\right)$ made opaque by adding powdered milk. A transparent Plexiglas platform (10 cm in diameter, $47 \mathrm{~cm}$ in height) was submerged $3 \mathrm{~cm}$ below the water surface and placed in one of the quadrants. The maze was located in the center of a well-lit room and surrounded by black curtains ( $50 \mathrm{~cm}$ from the pool periphery), which contained four distinct visual cues. The swimming path of each rat was monitored by an overhead video camera connected to a personal computer and analyzed by an automated tracking system (SmarTrack; Smartech, Madison, WI).

Spatial reference memory task. Before starting behavioral training, all rats were habituated to the water maze and observed for their swimming abilities by giving them a $90 \mathrm{~s}$ swim trial with no escape platform present. On the following day, they were subjected to the hidden platform version of the Morris water maze (Morris et al., 1982). The animals were required to locate a hidden platform that remained in the same position in relation to external visual cues during training days. The training was performed in blocks of three trials per day. To begin each trial, the rats were placed in the water, facing the maze wall, from one of three quadrants, except for the target quadrant containing the hidden platform. The daily order of entry into these quadrants was randomized. Each trial ended once the animals had found the platform; if the rats had not found the platform within $90 \mathrm{~s}$, they were guided there by an experimenter. After a period of $30 \mathrm{~s}$ on the platform, the rats were immediately replaced at a different start position for the next trial. At the end of a training session, the rats were dried and returned to their home cage.

Spatial working memory task. Whereas the water maze and its sur- rounding setup were the same in the spatial reference memory task, the delayed matching-to-place (DMP) version was used to assess the animals' spatial working memory (Steele and Morris, 1999). Throughout training, the escape platform was located $3 \mathrm{~cm}$ below the surface of the water in one of eight separate places within the pool (see Fig. $3 A$ ). The locations of the platform were altered from one day to the next in a pseudorandom manner, and the use of two locations in the same quadrant on two successive days was avoided. The DMP training lasted for $8 \mathrm{~d}$. The trials began at four equally spaced points (north, east, south, and west) in a randomized sequence with rats facing the wall. Four trials were given per day with an intertrial interval (ITI) of $5 \mathrm{~min}$, during which rats were kept in holding cages.

Spatial memory retrieval test. Probe trials were conducted $3 \mathrm{~h}$ after finishing daily training to evaluate the animals' retrieval performance of spatial memory under full-cue or partial-cue conditions (Nakazawa et al., 2002). The rats were released from the quadrant opposite to where the platform was and were allowed to swim for $90 \mathrm{~s}$ in the absence of the platform. We measured the percentage of time the rats spent in the area, which corresponded exactly to the area occupied by the platform during the training period. For the partial-cue probe trials, one cue that was located more distant from the platform was kept and the other three cues were removed from the surrounding curtains (Nakazawa et al., 2002).

Cue-place acquisition task. The rats were trained to swim to a visible platform, which was marked by a black and white striped plastic ball ( 5 $\mathrm{cm}$ in diameter, $10 \mathrm{~cm}$ high) and was raised $1 \mathrm{~cm}$ above the water surface. Four external cues were removed from the surrounding curtains to minimize interference from previously learned spatial information and to direct the animals' attention to the visible cue as much as possible. Training consisted of five trials with a $40 \mathrm{~min}$ ITI. For each trial, the location of the cued platform was moved to a different quadrant, and the start points were arranged so that the distance to the platform relative to the start point was constantly maintained across trials.

Intracranial microinjection. Muscimol (Sigma, St. Louise, MO), a GABA agonist, was dissolved in artificial CSF (aCSF), $\mathrm{pH} 7.2-7.4$, to a concentration of $0.5 \mu \mathrm{g} / 0.5 \mu \mathrm{l}$. Muscimol was chosen to reversibly inactivate $\mathrm{mPFC}$ without affecting the fibers of passage. A 33 gauge dual internal cannula (Plastics One) extending $1 \mathrm{~mm}$ below the end of the guide cannula was connected to a $10 \mu \mathrm{l}$ Hamilton syringe (Hamilton, Reno, NV) via polyethylene tubing (inner diameter, $0.38 \mathrm{~mm}$; outer diameter, $1.1 \mathrm{~mm}$ ), which was backfilled with distilled water. A small air bubble separated the water from the infusate. Either muscimol or aCSF was injected bilaterally at a rate of $0.15 \mu \mathrm{l} / \mathrm{min}$ using an infusion pump (model 101; KD Scientific, Holliston, MA). The injection quantity was $0.5 \mu \mathrm{l} /$ side (Lee and Kesner, 2003). The internal cannula was left in place for an additional 2 min to allow drug diffusion away from its tips. The rats were then returned to their home cages, and any abnormality in movement from the drug injection was carefully examined for $30 \mathrm{~min}$ before they were placed in the maze.

Fos immunohistochemistry and cell counting. Seventy minutes after training, rats were anesthetized with sodium pentobarbital $(60 \mathrm{mg} / \mathrm{kg}$, i.p.) and were perfused transcardially with $0.9 \% \mathrm{NaCl}$ followed by $4 \%$ paraformaldehyde in PBS, pH 7.4. Brains were then removed, postfixed overnight at $4^{\circ} \mathrm{C}$ in the same fixative, and transferred to $30 \%$ sucrose in PBS for cryoprotection. They were coronally cut (16 $\mu \mathrm{m}$ thick) with a cryostat (CM 3000; Leica, Nussloch, Germany). After washing freefloating sections in Tris buffered saline (TBS), they were pretreated with a solution containing $3 \%$ bovine serum albumin and $0.2 \%$ Triton $\mathrm{X}-100$ in TBS (TBST) for $60 \mathrm{~min}$ to block unspecific antibody binding. The sections were then incubated with primary rabbit anti-c-fos antibody (1:1000; Calbiochem, San Diego, CA) for $60 \mathrm{~min}$ at room temperature. After washing in TBST three times, the sections were placed in cyanine 3 (Cy3) goat-anti-rabbit IgG (1:1000; Invitrogen, Carlsbad, CA) containing blocking solution for $60 \mathrm{~min}$. The sections were rinsed in TBST three times and stained with 4',6'-diamidino-2-phenylindole (DAPI) (10 $\mu \mathrm{g} / \mathrm{ml}$ in TBS) for $10 \mathrm{~min}$ to counterstain nuclei. After washing, the sections were coverslipped with aqueous mounting medium (Biomeda, Foster City, CA).

For quantitative analysis, the regions of interest, $\mathrm{MPFC}$ and the hippocampus, were chosen because these structures are essential for pattern 
completion according to our lesion studies. Images of each region were obtained with an Axioskop 2 epifluorescent microscope (Zeiss, Thornwood, NY) equipped with a CoolSNAP HQ digital camera (Roper Scientific, Tucson, AZ) and MetaView software (Universal Imaging, West Chester, PA). The light level and camera exposure times against each sample were kept constant. Fluorescence was visualized with a UV filter (365 nm excitation) for DAPI and a green filter ( $546 \mathrm{~nm}$ excitation) for $\mathrm{Cy} 3$, and then two subsequent pictures were imported separately to Adobe Photoshop 7.0 (Adobe Systems, San Jose, CA) and superimposed. Three images $(200 \times 200 \mu \mathrm{m})$ per animal were taken for cell counting in PL, IL, CA1, CA3, the inner blade of dentate gyrus (iDG), and outer blade of dentate gyrus (oDG). Fos-immunoreactive (IR) cells were counted by an experimenter blind to the behavioral conditions. All neurons revealed by DAPI staining (blue) and Fos-IR cells revealed by Cy3 labeling (red) were counted. A neuron with Fos expression detected in its nucleus was considered as a Fos-IR cell because the immediate early genes function as transcription factors in the nucleus. We then calculated the ratio of Fos-IR neurons to DAPI-labeled cells per image. The percentages of three images were averaged as a mean and treated as $n=1$. Percentages for the following regions were combined as the pooled means: (1) mPFC, PL and IL; (2) DG, iDG and oDG.

Experimental procedures. In the first experiment, rats with CA3 lesions $(n=10)$, mPFC lesions $(n=11)$, and their sham operations (sham-CA3, $n=5$; sham-mPFC, $n=6$ ) were trained in the hidden platform version of the Morris water maze. Because there was no significant difference between the two sham-operated groups ( $p>0.5$ ), they were combined into one (sham). Performance of memory retrieval was examined using probe trials under full-cue (FC) or partial-cue (PC) conditions. After a 1 week rest period, three groups were subjected to the delayed matchingto-place version. Spatial working memory retrieval was then tested with three probe trials (first, second, and third: $\mathrm{P} 1, \mathrm{P} 2$, and $\mathrm{P} 3$, respectively). In the second experiment, we examined whether training experience in the partial-cue environment induced Fos expression in mPFC. Rats were initially trained to find a hidden platform with four distal cues for $6 \mathrm{~d}$ and then assigned to either FC $(n=8)$ or PC $(n=8)$ groups. On the final day, the FC group received the normal seventh training session, whereas the $\mathrm{PC}$ group was subjected to the training in the absence of three extramaze cues. Seventy minutes later, they were killed for evaluating Fos expression. To compare the number of Fos-IR neurons affected by nonmnemonic factors (Pace et al., 2005; Teather et al., 2005), two control groups were added. The naive $(n=4)$ group did not receive any training, whereas the yoked $(n=5)$ group was subjected to swimming in the maze with no platform for the duration of time comparable with the FC and the PC groups. In the third experiment, another set of rats with mPFC lesions $(n=11)$ and sham operations $(n=11)$ were trained in the hidden platform version in the single-cue environment so as to investigate whether contextual change disrupted the behavioral performance of mPFC-lesioned animals. Their spatial memory was evaluated in a probe trial under multiple-cue conditions. In the fourth experiment, we investigated whether $\mathrm{mPFC}$ inactivation disrupted spatial memory retrieval. Rats received training in the hidden platform version, and then probe trials were performed with infusions of either muscimol $(n=9)$ or aCSF $(n=9)$.

Histology. After the completion of the behavioral experiments, histological verification of lesion areas and cannula placements was performed. All rats were killed with an overdose of sodium pentobarbital ( 80 $\mathrm{mg} / \mathrm{kg}$ ) and were transcardially perfused with $0.9 \%$ saline, followed by a $10 \%$ formaldehyde solution. After extraction, the brains were postfixed in the same fixative overnight, transferred to $30 \%$ sucrose in $10 \%$ formaldehyde solution, and were stored at $4^{\circ} \mathrm{C}$ until they sank to the bottom. They were cut into $50 \mu \mathrm{m}$ coronal sections with a freezing microtome (SM 2000R; Leica). The sections then were mounted on gelatin-coated slides, stained with cresyl violet and coverslipped with Permount, and examined under light microscopy. To quantify the extent of electrolytic damage to the hippocampus and $\mathrm{mPFC}$, lesion boundaries were reconstructed using NIH ImageJ 1.38. The CA2 region was included in measurement of CA3 because CA2 resembles a terminal portion of CA3 (Gold and Kesner, 2005). The extent of lesions was expressed as the percentage of mean volume of each area in the sham group. Only rats with correct bilateral lesions (at least $70 \%$ of CA3 and mPFC) or with both cannula tips in $\mathrm{MPFC}$ were included in the data analysis.

Statistical analysis. For all experiments, group comparisons for escape latency, total distance, and swim speed were analyzed with repeatedmeasures ANOVA, followed by a Bonferroni's post hoc test, whereas group differences for target platform occupancy and mean Fos-IR counts were determined using Student's $t$ tests or one-way ANOVA with a Scheffés post hoc test. Differences of probe trials within the same group were compared with repeated-measures ANOVA. A Fisher's least significant difference post hoc test was conducted to determine whether each group searched significantly longer in the target platform area than in the other areas. All $t$ tests were two tailed. Only $p<0.05$ was considered to be statistically significant. All data were reported as means and SEM, unless specified otherwise.

\section{Results}

\section{The mPFC and CA3 lesions disrupt spatial memory retrieval} under partial-cue conditions

Using the hidden platform version of the Morris water maze, we evaluated the effects of the CA3 and mPFClesions on the animals' ability to acquire and retrieve spatial reference memory. The extent of lesions within each area is presented in Figure 1. A quantitative analysis revealed that $82.32 \pm 9.86 \%$ of CA3, $8.93 \pm$ $3.98 \%$ of CA1, and $3.87 \pm 3.81 \%$ of DG were damaged by the lesion targeted on CA3 (mean \pm SD). The mPFC lesion was centered on PL $(86.11 \pm 13.75 \%)$, including IL (42.72 $\pm 22.24 \%)$ and anterior portions of the Cg1 $(55.87 \pm 28.21 \%$, mean $\pm \mathrm{SD})$. The CA3, the mPFC, and the sham groups were trained to find a submerged platform under full-cue conditions. Over $10 \mathrm{~d}$, all groups required progressively less time to escape to the platform $\left(F_{(9,261)}=55.33, p<0.001\right)$ (Fig. $\left.2 A\right)$, and there were no significant differences in the escape latency and average swimming speed among the groups (no group effects for each measure, $\mathrm{F}_{(2,29)}<2.4, p>0.05$; no group $\times$ day interactions for each measure, $F_{(18,261)}<1.7, p>0.05$ ) (Fig. $2 A, C$ ). Total distance was also decreased gradually $\left(F_{(9,261)}=57.62, p<0.001\right)$ (Fig. $\left.2 B\right)$, but a significant effect of groups was observed $\left(F_{(2,29)}=4.07, p<\right.$ $0.05)$. A post hoc analysis (Bonferroni's procedure) revealed that the CA3 group moved a slightly longer distance than the mPFC group $(p<0.05)$. This difference might have resulted from an increased level of activity because of the hippocampal lesion (Teitelbaum and Milner, 1963; Godsil et al., 2005).

Probe trials were performed $3 \mathrm{~h}$ after finishing each regular training procedure on day $7(\mathrm{P} 1)$, day $9(\mathrm{P} 2)$, and day $10(\mathrm{P} 3)$ to access spatial memory retrieval by measuring the percentage time the rats spent in the platform area. On day 7 (P1), a repeatedmeasures ANOVA showed that all groups spent significantly more time in the target platform area than in the equivalent areas of the other three quadrants $\left(\mathrm{CA} 3, F_{(3,27)}=5.54, p<0.01\right.$; mPFC, $F_{(3,30)}=23.65, p<0.001$; sham, $F_{(3,30)}=12.54, p<$ 0.001 ) (Fig. $2 D, P 1$ ). The target platform occupancy also did not differ among groups $\left(F_{(2,29)}=0.07, p>0.9\right)$ (Fig. $\left.2 F, \mathrm{P} 1\right)$. Thus, neither CA3 nor mPFC lesion had any influence on the animals' ability to acquire and retrieve spatial reference memory.

After training 2 more days to prevent any extinction that may have occurred during the first probe trial, we conducted the second probe trial (P2) under the partial-cue condition in which all but one extramaze cue were removed from the surrounding curtains. The sham group displayed significantly higher occupancies of the phantom target platform position relative to other positions ( $\operatorname{sham,~} F_{(3,30)}=19.02, p<0.001$ ), whereas the CA3 and the $\mathrm{mPFC}$ groups failed to show such a preference $\left(\mathrm{CA} 3, F_{(3,27)}=\right.$ 2.37, $p>0.09$; mPFC, $F_{(3,30)}=2.45, p>0.08$ ) (Fig. $2 D, \mathrm{P} 2$ ). One-way ANOVA of the target occupancy time revealed a clear 
group effect $\left(F_{(2,29)}=6.18, p<0.01\right)$ (Fig. $2 F$, P2). A post hoc analysis (Scheffé's test) demonstrated that the partial-cue removal significantly disrupted the search preference of the CA3 and the mPFC groups compared with the sham group ( $p$ values $<0.05$ ).

To test whether the poor performance of the CA3 and the mPFC groups was attributable to oblivion of spatial memory, we restored the full-cue training environment and performed the third probe trial (P3). All groups searched significantly more in the target platform position than in any other platform position (CA3, $F_{(3,27)}=8.49, p<0.001 ; \mathrm{mPFC}, F_{(3,30)}=$ $10.09, p<0.001$; sham, $F_{(3,30)}=24.69, p<$ 0.001 ) (Fig. $2 D, \mathrm{P} 3$ ). The retrieval ability of the CA3 and the MPFC groups for the target platform was recovered as much as that of the sham group $\left(F_{(2,29)}=0.03, p>\right.$ 0.9 ) (Fig. 2F, P3), suggesting that faster memory loss cannot explain why the CA3 and the $\mathrm{mPFC}$ groups performed poorly in the partial-cue probe trial.

\section{mPFC is necessary for retrieving fast} acquired memory under partial-cue conditions but not for one-trial learning We next examined whether CA3 and mPFC lesions disrupted acquisition of novel platform locations in the DMP task, in which the location of the platform was
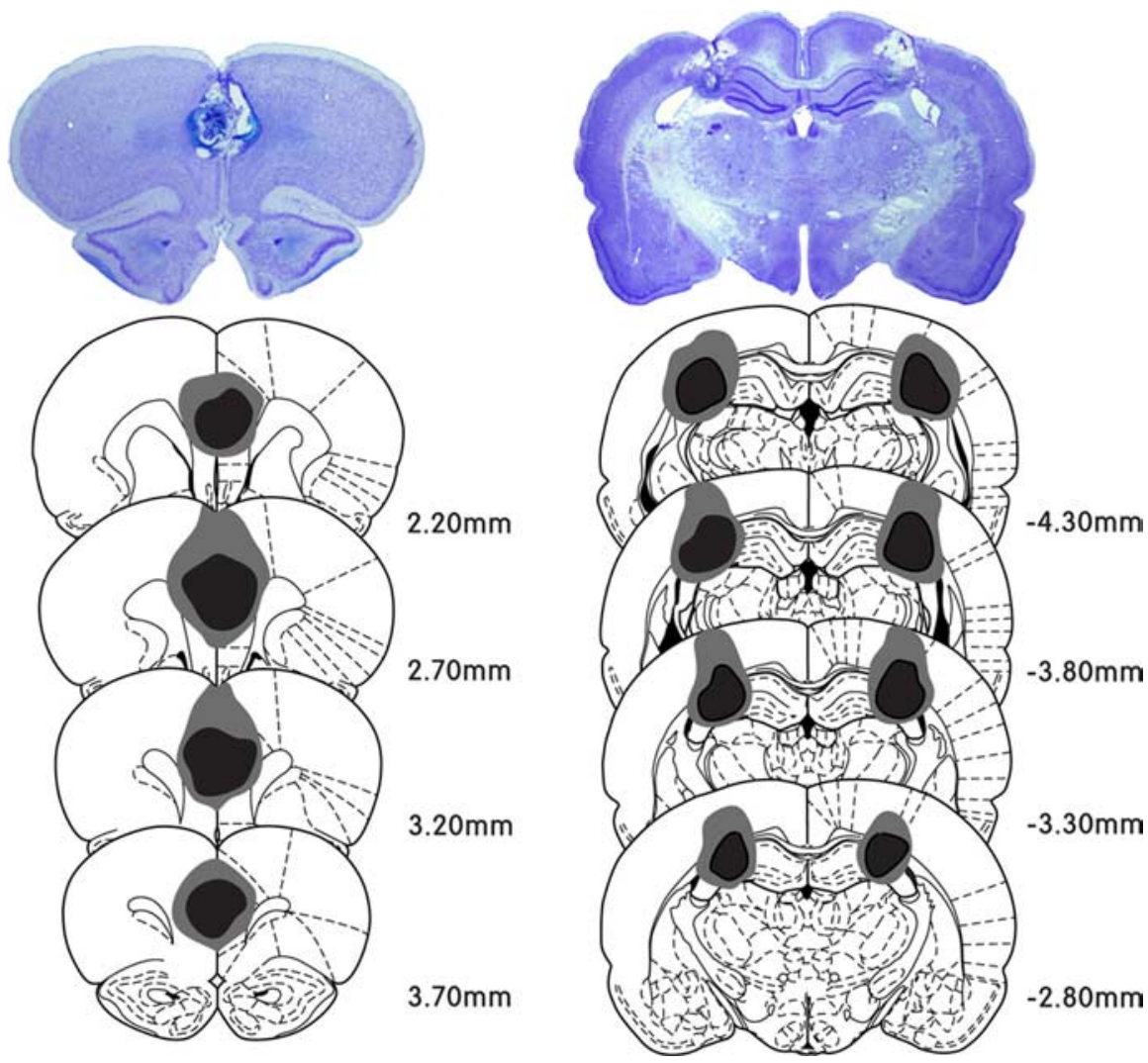

Figure 1. Histological evaluation of the extent of damage by electrolytic lesions in a series of sections of $m P F C$ and CA3. The photomicrograph shows a cresyl-violet-stained coronal section in each group. Illustrated below is the reconstruction of lesion sites of $\mathrm{mPFC}$ and CA3. The largest and the smallest lesioned areas are shown by gray and black, respectively. The anteroposterior stereotaxic coordinates for the sections are shown by the numbers beside the sections (modified from Paxinos and Watson, 1998). altered daily in a pseudorandom manner

(Fig. 3A). For data analysis and presentation, the time needed to locate the platform was measured, and the 8 DMP training days were subdivided into four blocks of 2 consecutive days. Because the animals had no previous knowledge of the platform location, the mean latencies for the first trials of each block were relatively long, but no difference was found among groups (block $1, F_{(2,61)}$ $=0.27, p>0.76$; block $2, F_{(2,61)}=2.05, p>0.14$; block $3, F_{(2,61)}$ $=1.58, p>0.21$; block $\left.4, F_{(2,61)}=0.51, p>0.6\right)$ (Fig. $\left.3 B\right)$. In the latencies of the second and subsequent trials, however, an ANOVA with a repeated-measures design showed significant group effect in blocks $1-3$ (block $1, F_{(2,61)}=7.53, p=0.001$; block 2, $F_{(2,61)}=16.81, p<0.001$; block 3, $F_{(2,61)}=3.59, p<$ 0.05). A post hoc analysis (Bonferroni's procedure) demonstrated that the mean latencies of the CA3 group were significantly longer than those of the sham and the mPFC groups in blocks 1 and $2(p$ values $<0.05$ ) and than that of the sham group in block 3 ( $p<$ $0.05)$. As the DMP training progressed, this impairment disappeared becuase there was no difference in the latencies of trials $2-4$ of block $4\left(F_{(2,61)}=1.47, p>0.2\right)$, suggesting that CA3lesioned animals can eventually learn the DMP task using an egocentric strategy rather than an allocentric one (Pearce et al., 1998). The latencies of trials 1 and 2 across the blocks are replotted in Figure 3C. A repeated-measures ANOVA revealed a significant group effect in the second trials $\left(F_{(2,61)}=12.067, p<0.001\right)$ but not in the first trials $\left(F_{(2,61)}=1.127, p>0.3\right)$. A post hoc analysis (Bonferroni's procedure) revealed that the CA3 group was longer in the escape latencies of the second trials than either the mPFC or the sham group ( $p$ values $<0.01$ ).

To assess the retrieval of fast acquired spatial memory, the first probe trial (P1) was given to all animals 5 min after the second trial of day 5 in the full-cue environment, which replaced the third trial. The mPFC and the sham group spent significantly more time in the target location relative to the corresponding locations in the nontarget quadrants (mPFC, $F_{(3,30)}=40.32, p<0.001$; sham, $\left.F_{(3,30)}=35.71, p<0.001\right)$, whereas the CA3 group did not show bias toward the target location $\left(F_{(3,27)}=1.17, p>0.33\right)$ (Fig. 3D, P1). Analysis of the target platform occupancy among groups revealed a significant group effect $\left(F_{(2,29)}=8.41, p=0.001\right)$ (Fig. 3F, P1). A post hoc analysis (Scheffé's test) demonstrated that the retrieval performance of the CA3 group was significantly lower than the performances of the mPFC and the sham groups ( $p$ values $<0.05$ ). These results suggest that the CA3 region is critical for acquiring memory of novel hidden platform locations in the DMP task (Nakazawa et al., 2003).

The three groups received another training session (day 6). On day 7, they were subjected to P2 5 min after the second trial under the partial-cue condition, which replaced the third trial. The CA3 and the mPFC groups did not search significantly more in the target area than in other platform zones (CA3, $\left.F_{(3,27)}=0.93, p>0.44 ; \mathrm{mPFC}, F_{(3,30)}=1.91, p>0.14\right)$, whereas the sham group maintained the search bias $\left(F_{(3,30)}=\right.$ 18.69, $p<0.001$ ) (Fig. 3D, P2). One-way ANOVA showed a significant group effect in the target platform occupancy $\left(F_{(2,29)}=9.48, p=0.001\right)$ (Fig. 3F). A post hoc analysis (Scheffé's test) demonstrated that the CA3 and the mPFC groups were impaired in the amount of time spent searching in the platform region relative to the sham group ( $p$ values $<0.01$ ), 
A
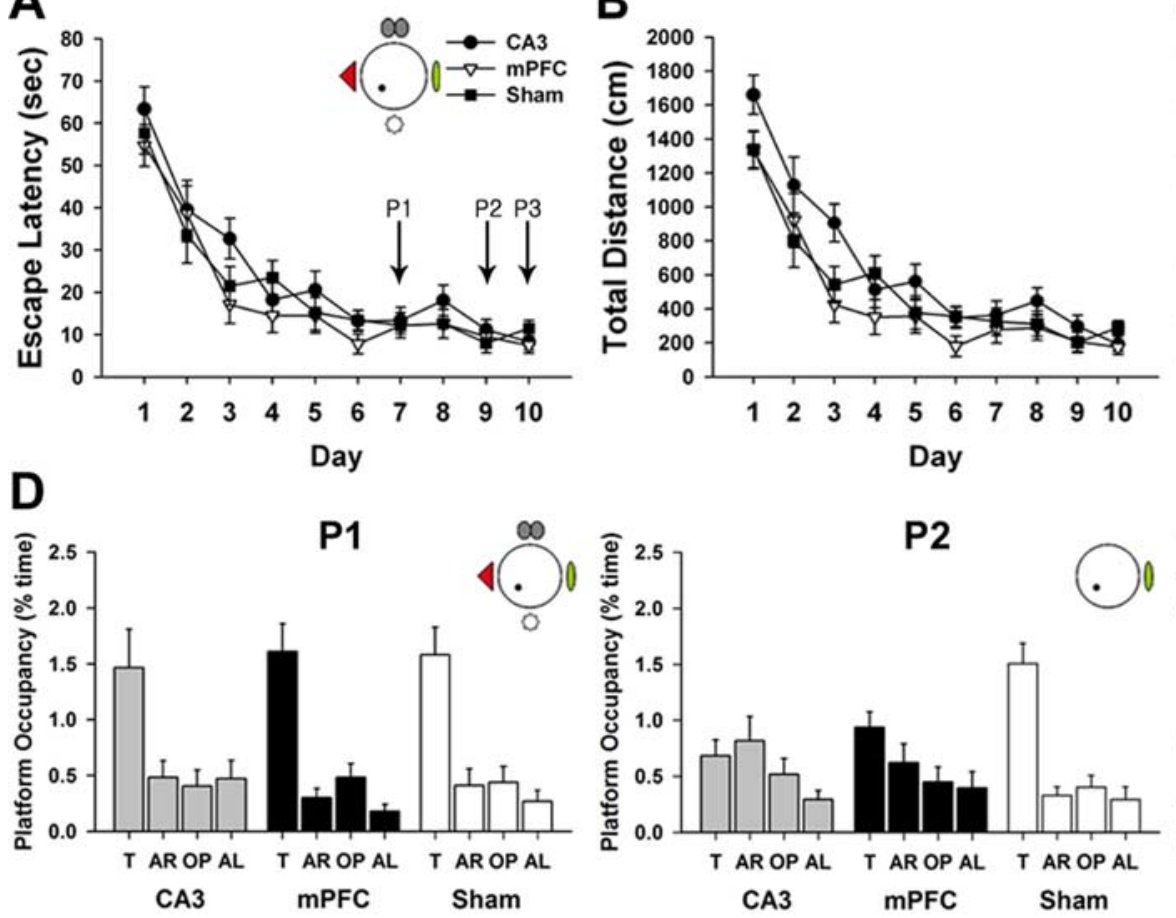

B

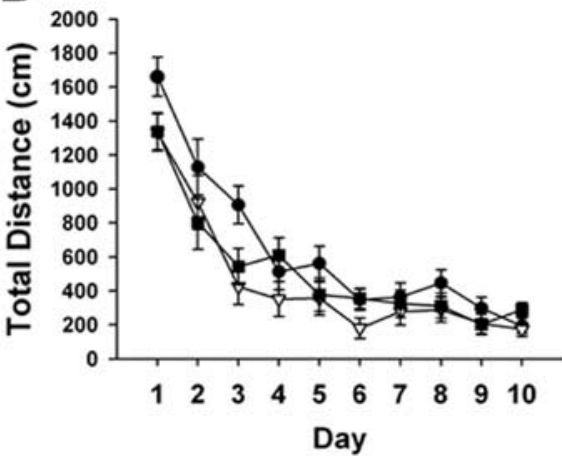

CA3
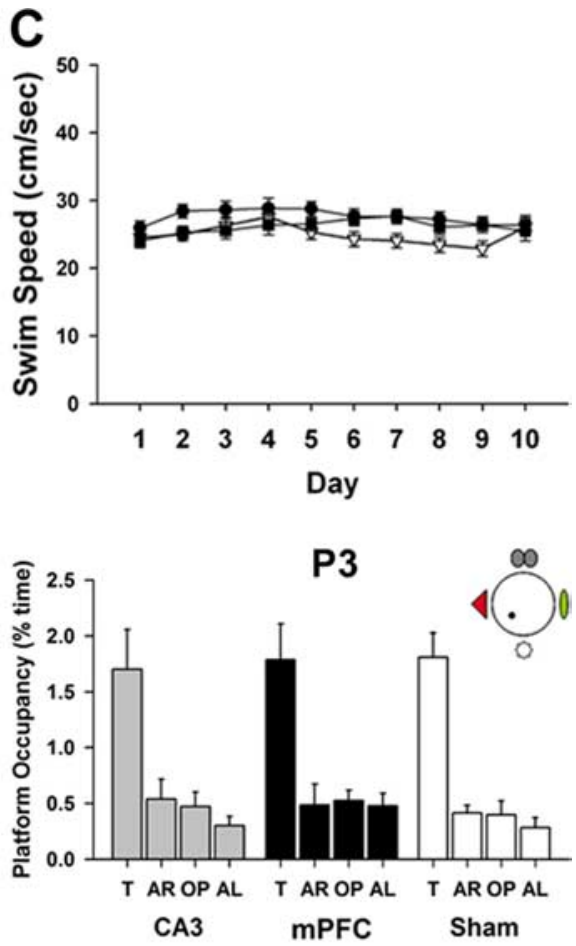

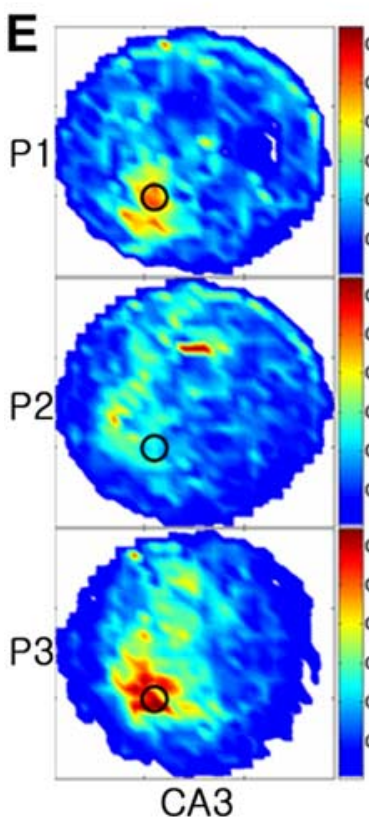

CA3

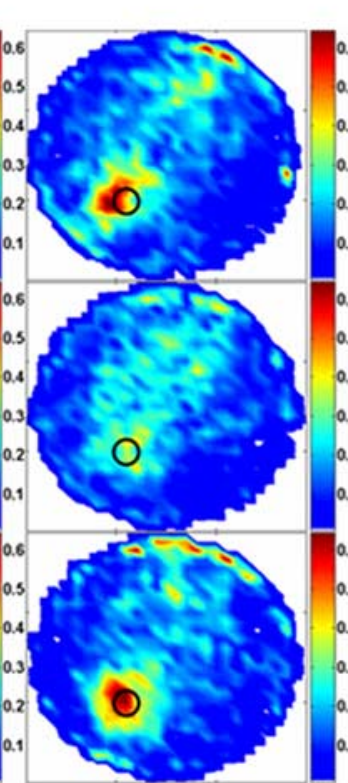

mPFC
$\mathbf{F}$

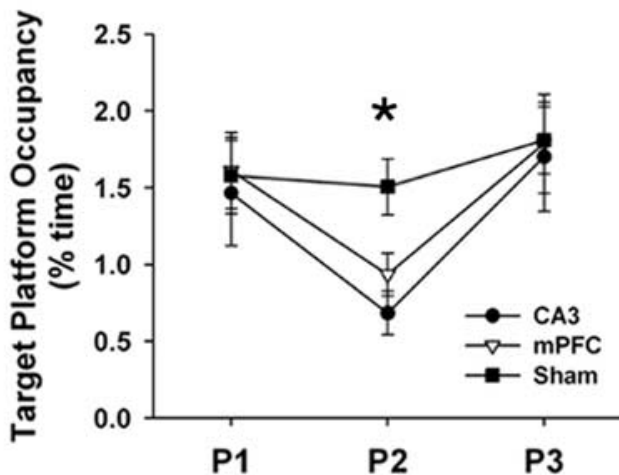

Figure 2. Effects of $\mathrm{mPFC}$ and $\mathrm{CA} 3$ lesions on retrieval of spatial reference memory. $A-C$, Rats were subjected to locate a hidden platform in the full-cue condition. The three groups did not differ significantly in escape latency $(\boldsymbol{A})$ or swimming speed (C). However, the CA3 group traveled longer than the mPFC group (B). D-F, Probe trials were given on day 7 (P1), day 9 (P2), and day $10(\mathrm{P} 3)$ to evaluate retrieval performance of each group under various conditions. In the full-cue conditions (P1, P3), all groups showed spatial preference for the target platform area (T) relative to the other areas [adjacent right (AR); opposite (OP); adjacent left (AL)], whereas in the partial-cue condition (P2), both the CA3 and the mPFC groups showed impaired retrieval performance (D). Spatial histograms of the animals' location during probe trials were illustrated $(\boldsymbol{E})$. To further elucidate the difference among groups, their target occupancy time was compared. The CA3 and the mPFC groups spent less time in the target platform than the sham group in $\mathrm{P} 2(\boldsymbol{F}) .{ }^{*} p<0.01$.

indicating that partial-cue removal disrupted the ability to retrieve spatial working memory in the $\mathrm{mPFC}$ group and that the CA3 group did not still acquire one-trial spatial memory appropriately.

On the next day, the third probe trial was given to test whether there was any delay-dependent deficit in spatial working memory retrieval. The animals received P3 60 min after the third trial of day 8 under the full-cue condition, which replaced the fourth trial. All groups except the CA3 group searched the target zone that had previously contained the escape platform more than other platform zones (CA3, $F_{(3,27)}=0.35, p>0.78 ; \mathrm{mPFC}, F_{(3,30)}$ $=9.04, p<0.001$; sham, $\left.F_{(3,30)}=14.22, p<0.001\right)$ (Fig. 3D, P3). Analysis of the target platform occupancy also revealed a significant group effect $\left(F_{(2,29)}=5.53, p<0.01\right)$ (Fig. 3F, P3). A post hoc analysis (Scheffe's test) indicated that the mPFC and the sham groups did not differ from each other and spent more time in the 
A

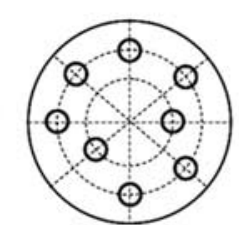

Platform locations

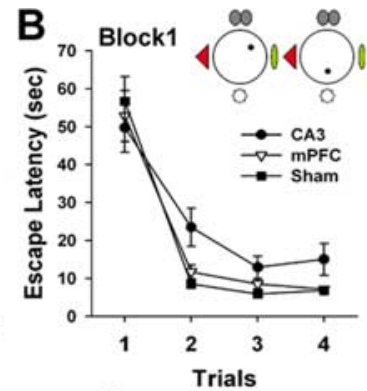

D P1
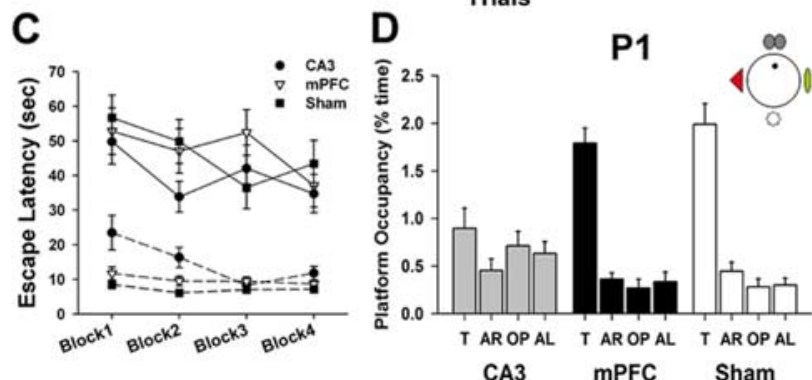

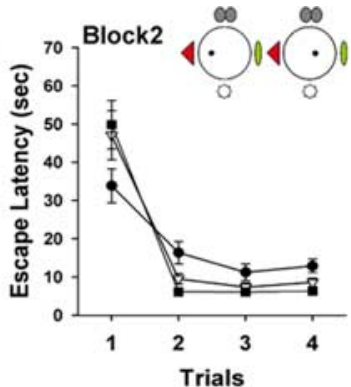

Trials
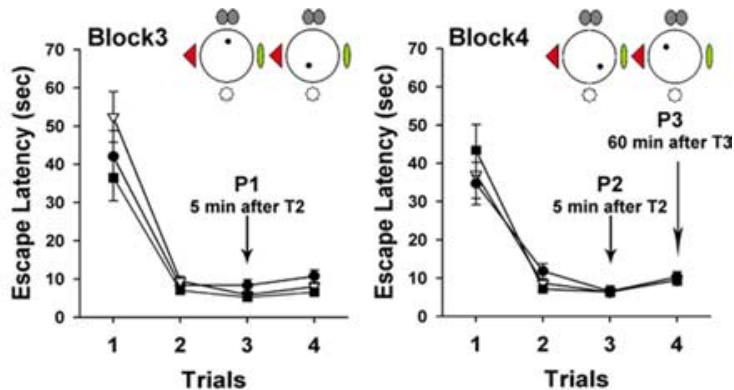

P2
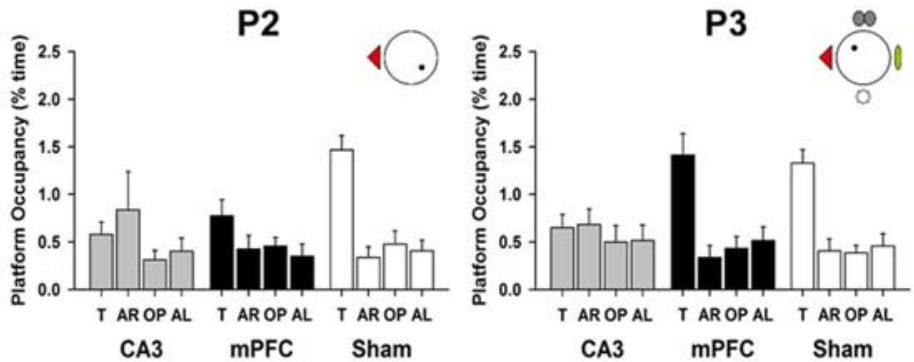

E

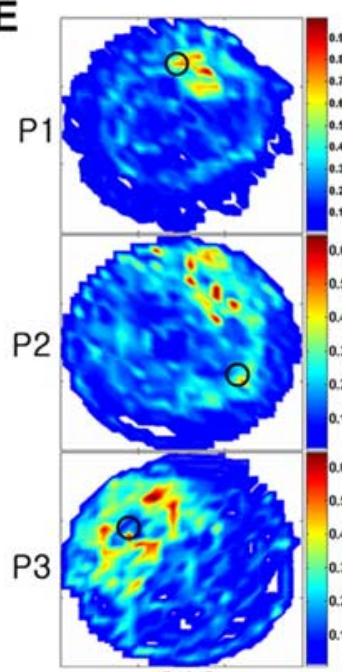

$\mathrm{CA3}$

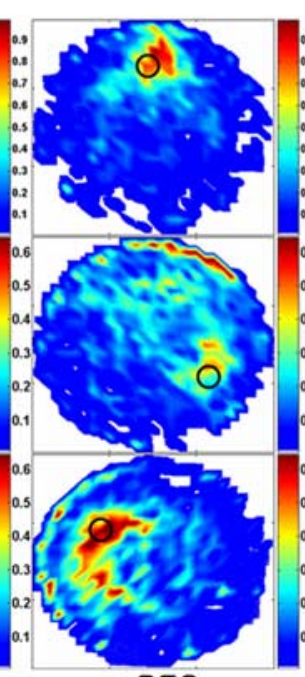

mPFC

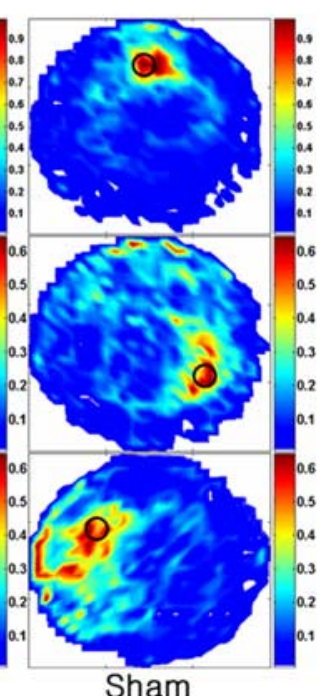

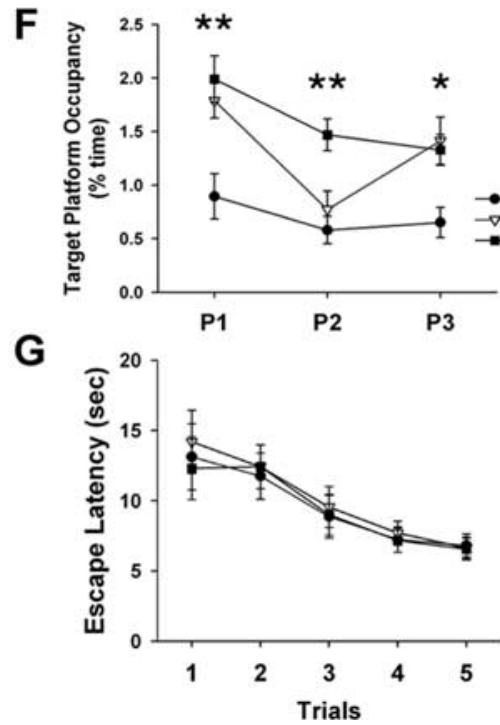

Figure 3. Effects of $\mathrm{mPFC}$ and CA3 lesions on retrieval of spatial working memory. $\boldsymbol{A}$, Eight platform locations (1 location per day) are displayed. $\boldsymbol{B}$, The three groups were trained in the DMP task for $8 \mathrm{~d}$, which were divided into four blocks of 2 days. Each trial was the average of two corresponding trials on consecutive days. The CA3 group required longer escape latencies in the second and subsequent trials of blocks 1-3, but mPFC-lesioned animals did not show any impairment across all blocks compared with the sham groups. C, Escape latencies of the first (solid lines) and second (dotted lines) trials during four training blocks were replotted. The CA3 group was impaired in the second trials but not in the first trials. D-F, Probe trials were performed on day 5 (P1), day 7 (P2), and day 8 (P3). The CA3 group did not display search bias toward the target areas in all probe trials. In contrast, the mPFC group spent more time in the platform areas (T) than in the other areas [adjacent right (AR); opposite (OP); adjacent left (AL)] under full-cue conditions, regardless of short-term (5 min, P1) and long-term (60 min, P3) delays. Only partial-cue removal compromised memory retrieval of the mPFC group (D). Spatial histograms of the animals' location during probe trials were depicted (E). The target platform occupancy for each group was further compared. The CA3 group spent less time in the target platform area than the sham group throughout all probe trials. The $\mathrm{mPFC}$ group searched significantly less in the target platform only in $\mathrm{P} 2(\boldsymbol{F}) .{ }^{*} p<0.01$, ${ }^{* *} p=0.001$. $\mathbf{G}$, In the visible platform task, there was no difference in escape latencies among groups.

phantom platform than the CA3 group ( $p$ values $<0.05$ ). These results, therefore, indicate that long-term delay (P3, $60 \mathrm{~min})$ as well as short-term delay (P1, 5 min) did not deteriorate the performance of the mPFC group, which tended to depend on the number of extramaze cues.

Seven days after finishing the spatial memory training, all groups were also trained in the visible platform version of the Morris water maze. There was no difference for learning cueplace association (trial effect, $F_{(4,116)}=12.45, p<0.001$; group effect, $F_{(2,29)}=0.17, p>0.8$ ) (Fig. $3 G$ ), which indicates that the three groups had a similar level of visual ability and motivation to find and climb onto the platform.
Partial-cue removal activates $\mathrm{MPFC}$ and CA3 for pattern completion in spatial learning

To investigate whether mPFC was involved in spatial memory retrieval in a degraded context, we next measured the expression of the immediate early gene c-fos, which is correlated with neuronal activation and is required for synaptic plasticity (Fleischmann et al., 2003). The FC and PC groups were subjected to the spatial reference memory task with four distal cues for $6 \mathrm{~d}$ so as to minimize Fos expression caused by novel and stressful experiences (Pace et al., 2005). Over training, the performance of the FC and $\mathrm{PC}$ groups improved in the escape latency and total distance (day effects for each measure, $F_{(5,70)}>69.56, p<0.001$ ) (Fig. 

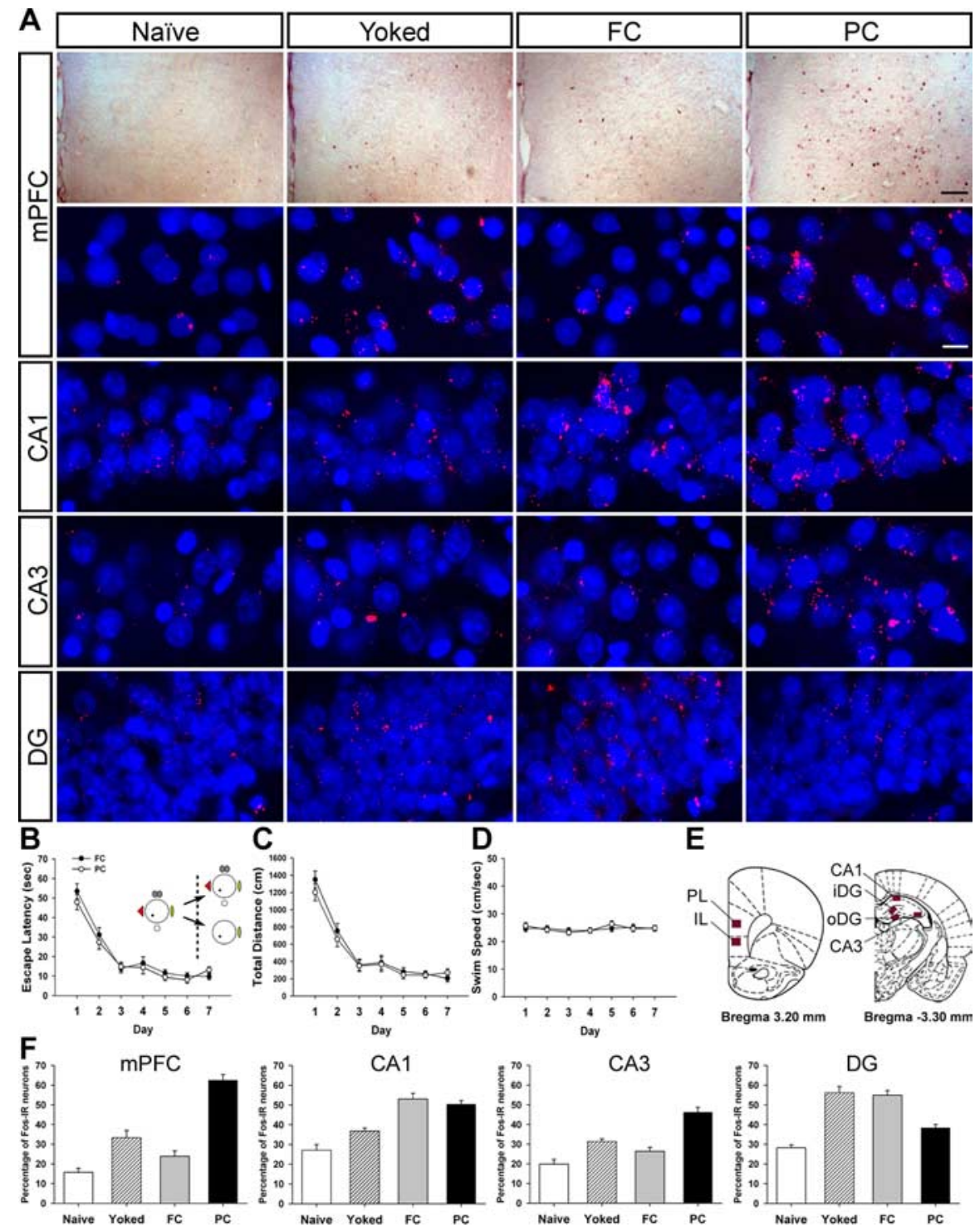

Figure 4. Fos expression in $\mathrm{mPFC}$ and the hippocampus after partial-cue removal. $\boldsymbol{A}$, Photomicrographs show that maze experience under various conditions differentially affected Fos expression. Gross Fos expression patterns in mPFC were identified using the avidin-biotin peroxidase method (top row). Fos expression in mPFC and the hippocampus was detected with a Cy3 fluorescent (red). Nuclei were stained with DAPI (blue) (bottom rows). Scale bars: top, $100 \mu \mathrm{m}$; bottom, $10 \mu \mathrm{m}$. $\boldsymbol{B}-\boldsymbol{D}$, The FC and the $P C$ groups were trained in the full-cue condition. There were no differences in escape latency $(\boldsymbol{B})$, distance traveled $(\boldsymbol{C})$, or swimming speed $(\boldsymbol{D})$. On the seventh day, the $P($ group received the training procedure with some cues removed, whereas the $F C$ group did the training procedure with the full set of cues. $\boldsymbol{E}$, Fos-IR neurons were examined in $\mathrm{MPFC}(\mathrm{PL}$ and IL), CA1, CA3, and DG (iDG and oDG). F, Training experience under degraded-cue conditions elevated Fos-IR neurons in mPFC and CA3.

nonmnemonic aspects such as swim stress, emotional arousal, and motor activity. As shown in Figure 4A, maze experience differentially affected Fos expression in both structures. One-way ANOVAs revealed significant main effects of group for the number of Fos-IR neurons in $\mathrm{mPFC}$ $\left(F_{(3,21)}=46.46, p<0.001\right), \operatorname{CA} 1\left(F_{(3,21)}=\right.$ 20.73, $p<0.001)$, CA3 $\left(F_{(3,21)}=24.60\right.$, $p<0.001)$, and DG $\left(F_{(3,21)}=28.55, p<\right.$ 0.001) (Fig. 4F). Scheffé's post hoc comparisons showed that the naive group exhibited a similar level of Fos expression to that of the FC group in mPFC ( $p$ value $>0.4$ ), to the yoked group in CA1 ( $p$ value $>0.1$ ), and to the yoked and the FC groups in CA3 ( $p$ values $>0.05$ ). In contrast to other studies in which Fos expression was evident in CA1 and CA 3 of yoked animals after massive water maze training in a single day (Guzowski et al., 2001; Teather et al., 2005), a similar level of Fos expression was found between the naive and the yoked groups in our study. This indicates that the prolonged training days minimized the number of Fos-IR neurons activated by nonmnemonic aspects of water maze training. In CA1, Fos expression of the FC and the PC groups was significantly greater than that of the naive or the yoked animals ( $p$ values $<0.01$ ). These data indicate that the animals trained to find a hidden platform showed more reliance on CA1 neurons than those who did not receive spatial training. In CA3 and mPFC, only the PC group that had experienced the water maze under partial-cue conditions showed significantly more Fos-IR neurons than other three groups ( $p$ values $<0.01$ ), whereas the FC group did not differ from the yoked group ( $p$ values $>0.2$ ), suggesting that both $\mathrm{mPFC}$ and CA3 contribute to spatial memory task with reduced allocentric information. As for DG, Fos expression of the FC and the yoked groups increased compared with that of the $\mathrm{PC}$ and the naive group ( $p$ values $<0.001)$. These findings imply that DG

$4 B, C)$, and there were no differences in the escape latency, total distance, and swimming speed between groups (no group effects for each measure, $F_{(1,14)}<1.27, p>0.2$; no group $\times$ day interactions or each measure, $F_{(5,70)}<0.89, p>0.3$ ) (Fig. $\left.4 B-D\right)$. On the seventh day, the FC group received the same procedure in the original full-cue environment, whereas the PC group was required to find the hidden platform under the reduced-cue condition (single cue). The PC group found the hidden platform as quickly as the FC group $\left(t_{(14)}=1.58, p>0.1\right.$ ) (Fig. $4 B$ ).

Seventy minutes after the completion of the final training, Fos expression in mPFC and the hippocampus was examined (Fig. $4 E$ ), because the ability to retrieve complete memory on the basis of incomplete cues seems to be essential for these brain structures, according to the previous lesion studies. Two control groups, the naive and the yoked groups, were used to control for might be involved in the recognition of a previously encountered environment, because only the FC and the yoked groups had been repeatedly exposed to the same environment over sessions (Lee and Kesner, 2002).

\section{Contextual change does not disrupt the performance of mPFC-lesioned animals}

Up to this point, partial-cue removal from four cues to one cue induced retrieval deficits in mPFC-lesioned animals and Fos expression in mPFC. It is, however, possible that simply changing the number of extramaze cues can cause such results (Ragozzino et al., 1999; Gisquet-Verrier and Delatour, 2006). We next tested, by using a new set of animals with $\mathrm{mPFC}$ lesion, whether changing the environment from one cue to four cues affected performance of spatial memory retrieval. A quantitative analysis re- 
vealed that electrolytic lesions produced $82.23 \pm 13.26 \%$ damage to PL, $39.82 \pm$ $31.23 \%$ damage to IL, and $58.17 \pm 34.44 \%$ damage to Cg1 (mean \pm SD) (Fig. $5 H$ ). Over the course of training, all animals showed an improvement in the time and the distance they took to find the escape platform (day effects for each measure, $F_{(9,180)}>34.6, p<0.001$ ) (Fig. 5A,B). Neither group differed in escape latency, total distance, or swimming velocity (no group effects for each measure, $F_{(1,20)}<$ $1.0, p>0.3$; no group $\times$ day interactions for each measure, $\left.F_{(9,180)}<1.36, p>0.2\right)$ (Fig. 5A-C).

To evaluate the effect of contextual change on spatial memory recall, two probe trials were performed on day 7 (P1) using the same training environment (single cue) and on day 9 (P2) with three distinct extramaze cues added (multiple cues). In the first probe trial, both groups showed a strong spatial bias toward the phantom area where the platform was located during training compared with the equivalent areas of the other three quadrants $\left(\mathrm{mPFC}, F_{(3,30)}=41.19, p<0.001\right.$; sham, $F_{(3,30)}=71.3, p<0.001$ ) (Fig. $5 D$, $\mathrm{P} 1)$, indicating that all groups acquired and recalled spatial reference memory well. For the second probe trial, which was given after 2 more training days to preclude any extinction from the first probe trial, the mPFC and the sham groups also searched significantly longer in the platform area than in other areas $(\mathrm{mPFC}$, $F_{(3,30)}=29.86, p<0.001$; sham, $F_{(3,30)}=$ 20.79, $p<0.001$ ) (Fig. 5D, P2). Analysis of the target platform occupancy between groups also revealed that the mPFC group exhibited similar retrieval performance to the sham group in both probe trials ( $\mathrm{P} 1$, $t_{(20)}=0.96, p>0.3 ; \mathrm{P} 2, t_{(20)}=0.28, p>$ 0.7 ) (Fig. $5 E$ ). Furthermore, a paired $t$ test showed that the platform occupancy under single-cue conditions was comparable with that under multiple-cue conditions in both the $\operatorname{mPFC}\left(t_{(10)}=1.6, p>0.1\right)$ and the sham $\left(t_{(10)}=1.03, p>0.3\right)$ groups. Therefore, these results indicate that contextual change, as long as the original context is not degraded, does not disrupt retrieval performance of mPFC-lesioned animals.

\section{Inactivation of mPFC disrupts retrieval of spatial memory in the partial-cue condition}

We next determined whether temporal inactivation of $\mathrm{mPFC}$ before retrieval tasks had the same effect as permanent mPFC lesions. Rats with guide cannulas aimed at mPFC (Fig. $6 H$ ) were trained for $12 \mathrm{~d}$ to locate a hidden platform in the environment containing four extramaze cues. During training, the time and distance to reach the platform decreased significantly (day effects
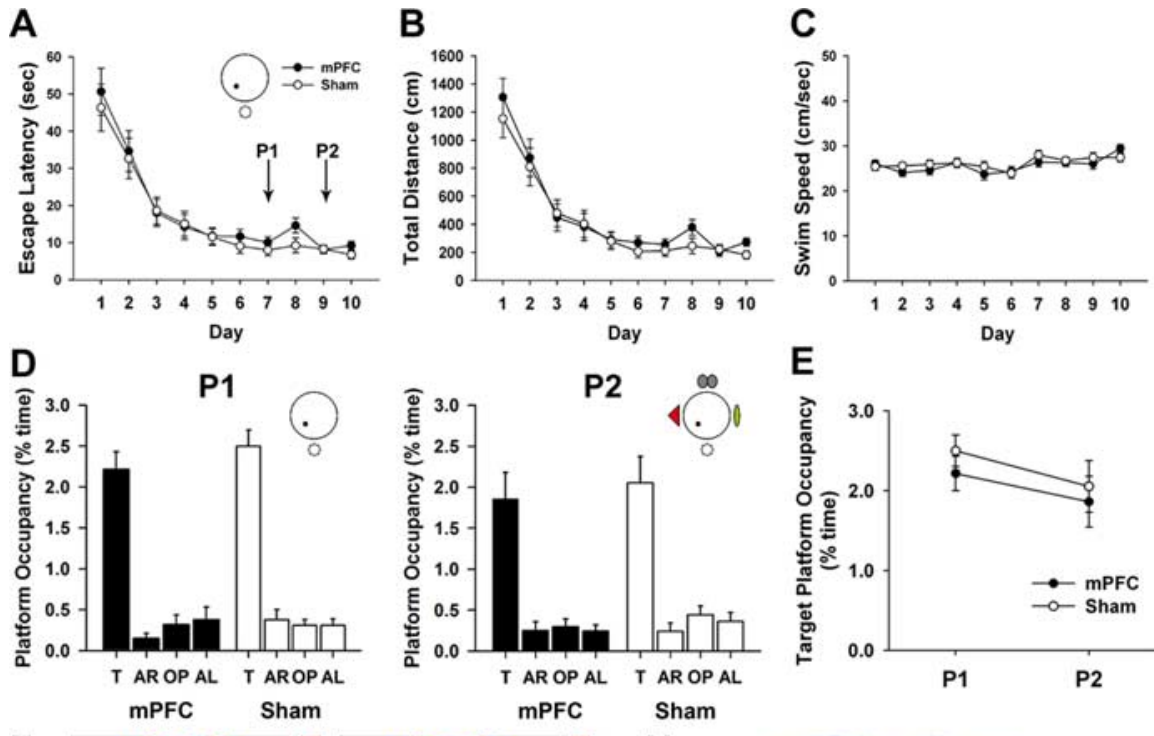

E

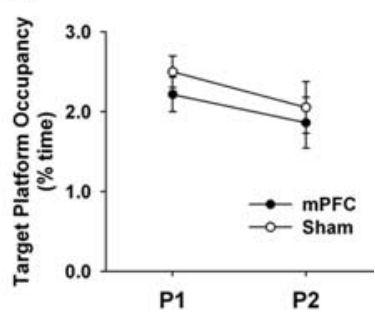

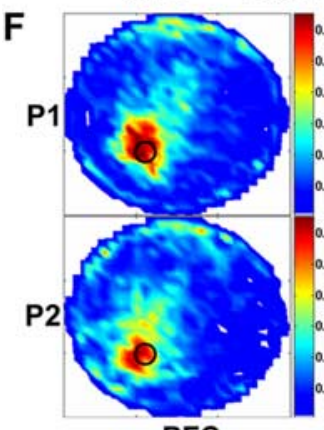

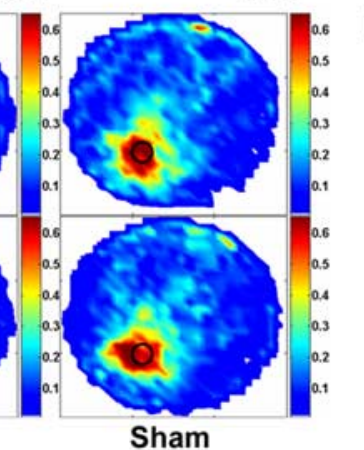

H
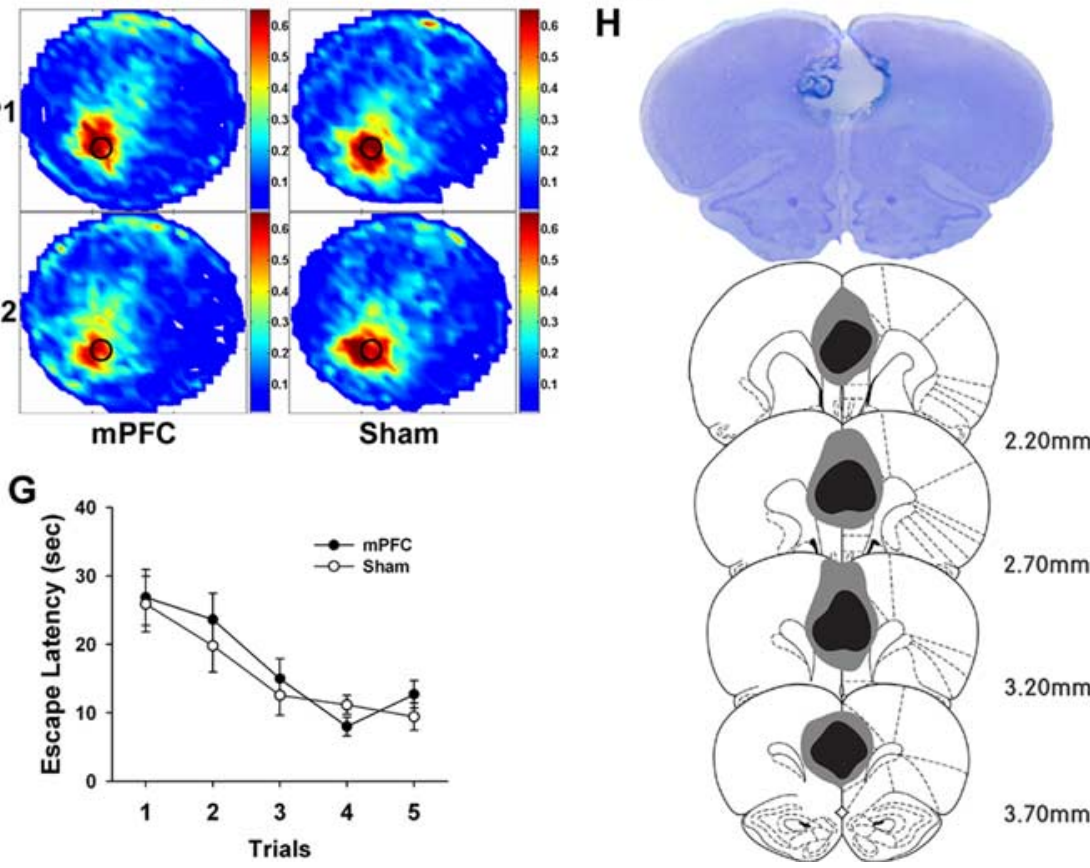

Figure 5. Effects of contextual change on retrieval performance of mPFC-lesioned animals. A-C, Animals were trained to locate a hidden platform in the single-cue environment. The $\mathrm{MPFC}$ and the sham groups acquired spatial reference memory at essentially the same rate. No differences were observed in escape latency $(\boldsymbol{A})$, distance traveled $(\boldsymbol{B})$, or swimming speed $(\boldsymbol{C})$. D $\boldsymbol{F}$, Two probe trials were given on day 7 (P1) and day 9 (P2). The mPFC-lesioned animals showed robust spatial bias toward the target area (T) compared with the other areas [adjacent right (AR); opposite (OP); adjacent left (AL)], even in the presence of extra cues in P2 (D). The target platform occupancy of two probe trials did not differ between groups $(\boldsymbol{E})$. Spatial histograms of the animals' location during probe trials were illustrated $(\boldsymbol{F}) . \mathbf{G}$, In the visible platform task, both groups acquired the stimulus-response associations. $\boldsymbol{H}$, The extent of electrolytic lesions in MPFC mapped onto coronal rat brain sections (adapted from Paxinos and Watson, 1998). The photomicrograph shows a cresyl-violet-stained section. The largest and the smallest lesioned areas are displayed by gray and black, respectively.

for each measure, $F_{(11,176)}>45.75, p<0.001$ ) (Fig. $6 A, B$ ), and no differences were observed in the escape latency, total distance, and swimming speed between groups (no group effects for each measure, $F_{(1,16)}<2.05, p>0.1$; no group $\times$ day interactions for each measure, $F_{(11,176)}<1.27, p>0.2$ ) (Fig. $6 A-C$ ).

Retrieval performance was evaluated using probe trials. On day 5 (P1), rats were infused with muscimol or aCSF into mPFC. After $30 \mathrm{~min}$, the first probe trial was conducted in full-cue conditions. All groups searched significantly more time in the platform area than in other areas (muscimol, $F_{(3,24)}=10.68, p<$ 
A

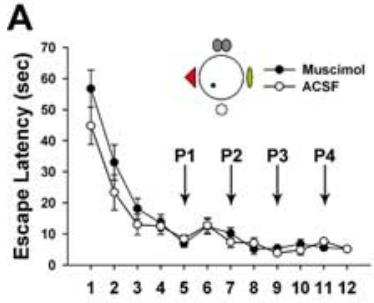

Day
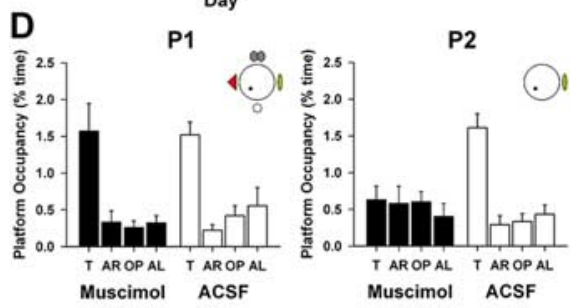

B

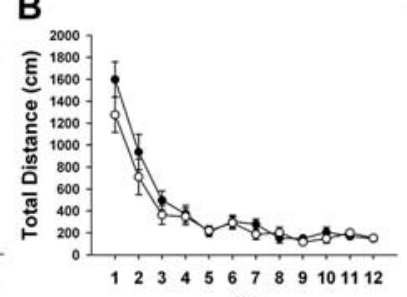

Day
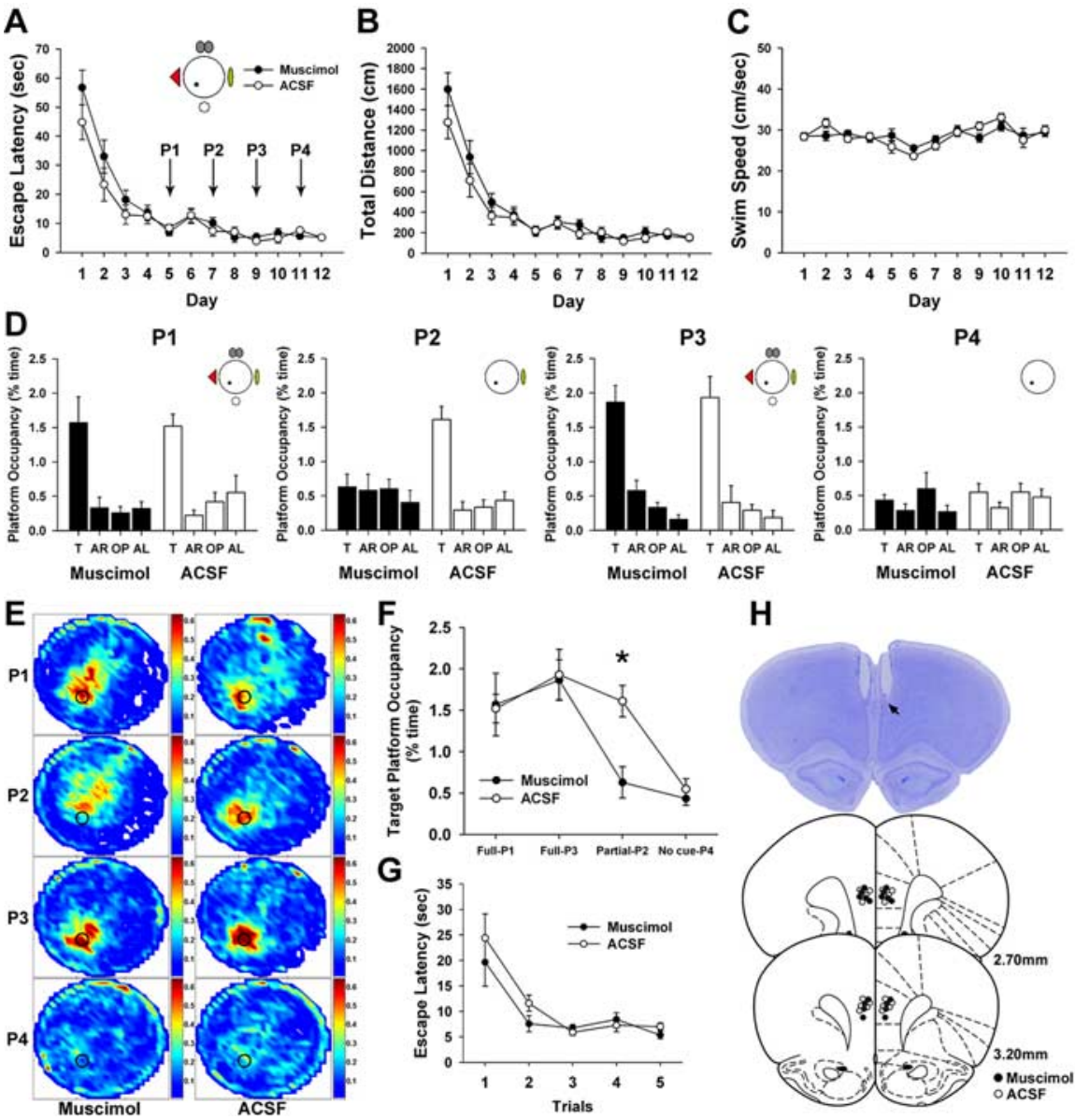

$F$

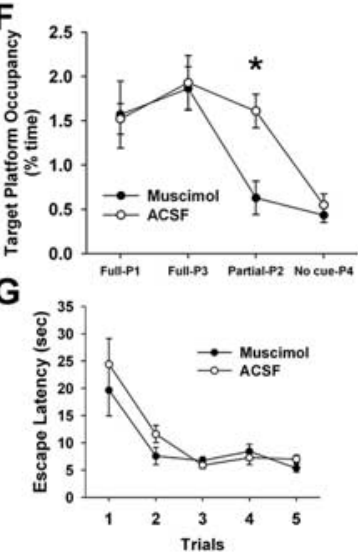

H

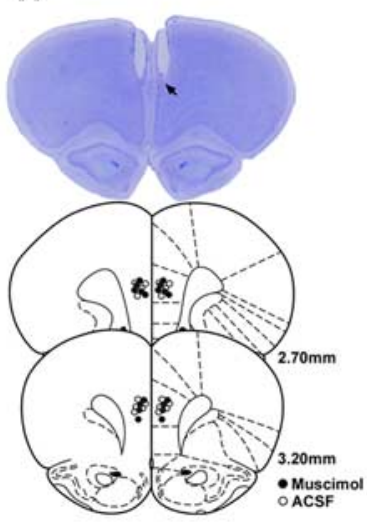

Figure 6. Effects of muscimol inactivation of mPFC on spatial memory retrieval. $\boldsymbol{A}-\boldsymbol{C}$, Rats were trained to acquire spatial reference memory in the full-cue condition. The two groups did not differ significantly in escape latency $(\boldsymbol{A})$, total distance $(\boldsymbol{B})$, or swimming speed (C). D, Probe trials were performed on day 5 (P1) and day 7 (P2). Inactivation of mPFC did not impair retrieval performance in the full-cue condition (P1). However, mPFC inactivation disrupted spatial preference for the target platform (T) relative to the other areas [adjacent right (AR); opposite (OP); adjacent left (AL)] under incomplete-cue condition (P2). Furthermore, to investigate reliance on distal cues for spatial memory retrieval, two more probe trials were conducted in drug-free states on day 9 (P3) and day 11 (P4). Removal of all extramaze cues disrupted retrieval performance of both groups. $\boldsymbol{E}$, Spatial histograms of the animals' location during probe trials were illustrated. $\boldsymbol{F}$, The target platform occupancy of probe trials was displayed. Inactivation of mPFC disrupted search preference for the target platform under partial-cue conditions. ${ }^{*} p<0.01$. G, In the visible platform task, both groups acquired the cue-place associations. $\boldsymbol{H}$, Cannula placements in mPFC were evaluated. The photomicrograph shows a cresyl-violet-stained section. The arrow indicates the tip of the injection cannula. Illustrated below is the location of microinjection sites for all animals included in analysis (modified from Paxinos and Watson, 1998).

0.001; aCSF, $F_{(3,24)}=12.39, p<0.001$ ) (Fig. $6 D$, P1), suggesting that $\mathrm{mPFC}$ inactivation did not impair retrieval ability when all extramaze cues were available. After 2 training days, rats were treated with muscimol or aCSF and received the second probe trial (P2) under partial-cue conditions. Inactivation of mPFC disrupted search preference for the target platform $\left(F_{(3,24)}=\right.$ $0.33, p>0.8$ ), whereas aCSF-infused rats displayed significantly higher occupancies of the target zone relative to other zones $\left(F_{(3,24)}=20.97, p<0.001\right)$ (Fig. $\left.6 D, \mathrm{P} 2\right)$. Independent $t$ tests showed a significant difference in the target platform occupancy between groups under partial-cue (P2), but not full-cue (P1), conditions $\left(\mathrm{P} 1, t_{(16)}=0.12, p>0.9 ; \mathrm{P} 2, t_{(16)}=3.66, p<0.01\right)$ (Fig. 6F). Although the muscimol-infused rats seemed to be impaired in finding the exact target location after partial-cue removal, their tendency to focus navigation within a certain location was not completely disrupted. Their search pattern was concentrated in the place slightly above the target platform location (Fig. 6E, P2). If a phantom platform of the same size is
Day

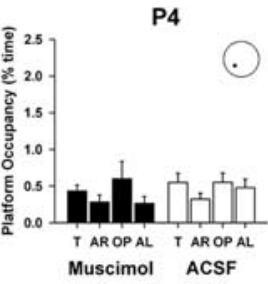

shifted toward the center of the most occupied area, then the difference between the muscimol- and aCSF-infused group disappears (muscimol, $1.59 \pm 0.36$; aCSF, $\left.1.61 \pm 0.19 ; t_{(16)}=0.05, p>0.9\right)$, suggesting that $\mathrm{MPFC}$ inactivation disturbs search accuracy.

\section{Retrieval performance depends on spatial cues}

To investigate whether the retrieval in the partial-cue environment relied on the remaining distal cues surrounding the maze, we performed two more probe trials in a drug-free state on day 9 (P3) with four distal cues present and on day 11 (P4) with all visual cues removed. When four distal cues were available, both groups concentrated their search in the target platform area relative to other areas (muscimol, $F_{(3,24)}=29.2, p<0.001 ; \mathrm{aCSF}, F_{(3,24)}=$ $16.8, p<0.001$ ) (Fig. $6 D$, P3). In contrast, neither group searched selectively in the target area when all extramaze cues were absent in $\mathrm{P} 4$ (muscimol, $F_{(3,24)}=1.14, p>$ 0.35 ; aCSF, $F_{(3,24)}=1.2, p>0.32$ ) (Fig. $6 D, \mathrm{P} 4)$. We then determined whether this poor retrieval performance was attributable to deficits in motivation, motor coordination, or vision. When animals were trained in the visible platform version, both groups acquired strong stimulus-response association $\left(F_{(1,16)}=1.11, p>0.3\right)$ (Fig. 6G). These results suggest that animals use allocentric representation of the distal cues to locate the escape platform and that retrieval deficits result from the lack of spatial information.

\section{Discussion}

The present study was undertaken to determine which brain structures were crucial for spatial memory recall after partialcue removal. Lesion and inactivation experiments demonstrated that both CA3 and $\mathrm{mPFC}$ were indispensable for spatial pattern completion. Expression of Fos-IR neurons corroborated these behavioral data: experience of additional training in the absence of all except one cue increased the number of Fos-IR cells in $\mathrm{mPFC}$ and CA3. Together, these findings provide compelling evidence that interactions between $\mathrm{mPFC}$ and the hippocampus become essential for spatial memory retrieval once allocentric information is reduced.

\section{CA3 and spatial memory acquisition}

Spatial learning in the hidden platform task was unaffected by CA3 lesions, suggesting that reference memory acquisition over multiple sessions can be achieved by other brain areas. Considering the pattern of elevated Fos expression in animals trained to find a fixed location relative to extramaze cues (Fig. $4 F$ ), spatial reference memory needs neural activity in CA1 (Tsien et al., 1996). Conversely, CA3 lesions produced severe deficits in acquisition of the DMP task, which demands animals to rapidly incor- 
porate the information of a novel location into spatial representations of the environment. Such poor encodings likely lead to retrieval failure of CA3-lesioned animals during probe trials regardless of the number of extramaze cues (Nakazawa et al., 2003). The data showing differential involvement of CA1 and CA3 are consistent with several studies that have established hippocampal subregion-specific functions in spatial learning: CA1 is necessary for acquisition of spatial reference memory, whereas CA3 is required for spatial working memory or one-trial learning by disambiguating changes in the environment (Moser and Moser, 1998; Lee and Kesner, 2002; Leutgeb et al., 2004; Nakazawa et al., 2004).

In addition, CA3-lesioned animals were impaired to recall spatial reference memory when the extramaze cues were degraded to the partial-cue condition but performed normally under full-cue conditions. Consistent with the behavioral data, training in the absence of some of the original cues elevated the number of Fos-IR neurons in CA3. These support the theory that, when cues are fragmented, CA3 memory trace is restored by synaptic modification of recurrent connections in a NDMA receptor-dependent manner, which in turn activates CA1 neurons to retrieve the complete set of spatial reference memory (Marr, 1971; Treves and Rolls, 1994; Nakazawa et al., 2002). Thus, combined with previous studies on the CA3 network, our results from two spatial memory tasks strongly suggest that rapid formation of tight ensemble patterns in CA3 underlies the ability to acquire spatial memory in a single trial as well as the ability to maintain homologous output in the presence of degraded inputs (Lee et al., 2004; Nakazawa et al., 2004; Leutgeb et al., 2006).

\section{mPFC and spatial memory retrieval}

Contrary to the CA3 group, mPFC-lesioned animals learned and recalled both spatial reference and working memory in the fullcue environment normally. Interestingly, no disruption was obtained when they were tested to retrieve rapidly acquired locations after 5 and 60 min delay periods. This is inconsistent with previous studies that the rodent $\mathrm{mPFC}$ is engaged in spatial working memory (Delatour and Gisquet-Verrier, 1996; Ragozzino et al., 1998; Porter et al., 2000). However, this idea has been recently challenged by observations that $\mathrm{MPFC}$ is not directly involved in the short-term maintenance of information but rather in behavioral flexibility and strategy shifting (Ragozzino et al., 1999; Delatour and Gisquet-Verrier, 2000). Our results strongly indicate that mPFC does not contribute to the temporary storage of spatial information (Rowe et al., 2000; Gisquet-Verrier and Delatour, 2006) and that animals with the intact hippocampus can maintain spatial working memory, regardless of the integrity of mPFC (Lee and Kesner, 2003). Conflicting findings may be attributable to differences in testing procedures, lesion methods, and/or lesion locations. Especially with respect to testing procedures, most of the studies used a delayed non-matching-to-place task with positive reinforcement, such as the radial arm maze, whereas we used a delayed matching-to-place procedure that involves escape from aversive stimulation.

Only when the number of available cues was reduced did mPFC-lesioned animals exhibit retrieval deficits constantly in the reference and the working memory tasks. Compatible with the lesion effect, challenging normal animals to find the target platform under partial-cue conditions induced Fos expression in mPFC. Closer examination using pharmacological inactivation revealed that $\mathrm{mPFC}$ inactivation before probe trials impaired retrieval performance under partial-cue, but not complete-cue, conditions. Because the animals were able to acquire spatial memory with intact mPFC, this effect cannot be attributed to the encoding deficit. Thus, $\mathrm{mPFC}$ is crucial for retrieval and utilization of stored memory when faced with modified cues. It is plausible that such retrieval deficits may be attributable to the contextual change per se, because previous studies report that $\mathrm{mPFC}$ is engaged in shifting behavioral strategies when changes occur within the experimental procedures (Gisquet-Verrier and Delatour, 2006) and in extinction of learned fear memory, in which the behavioral responses need to be adapted to new contingencies (Quirk et al., 2000). However, changing the environment from single cue to multiple cues did not affect retrieval performance of mPFC-lesioned animals, suggesting that a degraded input, rather than enriched one, requires $\mathrm{mPFC}$ to retrieve spatial memory. Together, our results indicate that $\mathrm{mPFC}$ contributes to spatial pattern completion through memory retrieval rather than encoding or strategy shifting.

Two feasible mechanisms by which pattern completion might be attained are suggested here. The first hypothesis is based on robust reciprocal connections between $\mathrm{MPFC}$ and the entorhinal cortex (EC), the major cortical input to the hippocampus (Eichenbaum, 2000; Vertes, 2006). Such anatomical arrangement suggests that $\mathrm{mPFC}$ input to the hippocampus might be critical for spatial pattern completion. Electrophysiological experiments have found that sensory input for an environment is initially transformed into allocentric spatial representation in EC and then subsequently stored in the hippocampus (Fyhn et al., 2004; Hafting et al., 2005). In this circuit, mPFC receiving convergent input from multiple brain regions (Conde et al., 1995) would help EC to develop a spatial map with its integrated information. This hypothesis is supported by observations that mPFC neurons are capable of monitoring spatial information (Hok et al., 2005) and that $\mathrm{mPFC}$ lesions alter hippocampal place cell activity after a long-term delay (Kyd and Bilkey, 2003). Thus, in the full-cue environment, the visual cortex may provide EC with robust allocentric input, sufficient to recall the whole spatial memory regardless of mPFC (Nakazawa et al., 2002). In contrast, under partial-cue conditions, limited sensory input might be too weak to trigger the entire memory without $\mathrm{MPFC}$ and consequently retard retrieval. In our experiments, $\mathrm{mPFC}$ inactivation before partial-cue probe trials did perturb search accuracy but did not compromise coherent navigation behavior concentrated on an area slightly off the target platform location (Fig. 6E, P2). These data support the role of $\mathrm{mPFC}$ input to the hippocampus in spatial pattern completion.

Alternatively, mPFC might be involved in retrieving memory from the hippocampus, when it becomes degraded. In support of this, $\mathrm{mPFC}$ (PL/IL) contributes to remote memory retrieval after more than a 30 d delay (Frankland et al., 2004; Maviel et al., 2004), during which remote memory can be easily degraded because of interference introduced by new experience (Biedenkapp and Rudy, 2007). Our results also show that mPFC is necessary for spatial memory retrieval when the spatial cues are degraded. Under partial-cue conditions, mPFC may be engaged in integrating the less precise memory provided by the hippocampus with other inputs from neocortical systems, synthesizing it into a more complete form to navigate to a goal location (Treves and Rolls, 1994; Hok et al., 2005). Interestingly, it has been suggested that the nucleus accumbens (NA), another major output structure of the hippocampus, is necessary for spatial memory processes (Floresco et al., 1997; Thierry et al., 2000). However, electrolytic lesions of NA did not impair spatial learning in a reference memory task or memory retrieval in the partial-cue environments (data 
not shown), suggesting that the hippocampal output through mPFC, but not through NA, is critical for pattern completion.

In summary, spatial memory retrieval from fragments of original cues involves contributions from several brain areas. At the core lies CA3, which stores memory as a pattern of activation among interconnected neurons and reactivates spatial memory stored in CA1 (Nakazawa et al., 2002; Lee et al., 2004). The current study also identified $\mathrm{mPFC}$ and its interaction with CA3 as the key element in spatial pattern completion. We propose that $\mathrm{mPFC}$ plays a significant role in retrieving spatial memory by providing a complementary input into the hippocampus and/or by improving spatial information from the hippocampus into a more precise form. Increased mnemonic demand, such as reduced allocentric cues, seems to call for a synergistic interaction between the hippocampus and mPFC.

\section{References}

Biedenkapp JC, Rudy JW (2007) Context preexposure prevents forgetting of a contextual fear memory: implication for regional changes in brain activation patterns associated with recent and remote memory tests. Learn Mem 14:200-203.

Burette F, Jay TM, Laroche S (1997) Reversal of LTP in the hippocampal afferent fiber system to the prefrontal cortex in vivo with low-frequency patterns of stimulation that do not produce LTD. J Neurophysiol 78:1155-1160.

Conde F, Maire-Lepoivre E, Audinat E, Crepel F (1995) Afferent connections of the medial frontal cortex of the rat. II. Cortical and subcortical afferents. J Comp Neurol 352:567-593.

Delatour B, Gisquet-Verrier P (1996) Prelimbic cortex specific lesions disrupt delayed-variable response tasks in the rat. Behav Neurosci 110:1282-1298.

Delatour B, Gisquet-Verrier P (2000) Functional role of rat prelimbicinfralimbic cortices in spatial memory: evidence for their involvement in attention and behavioural flexibility. Behav Brain Res 109:113-128.

Eichenbaum H (2000) A cortical-hippocampal system for declarative memory. Nat Rev Neurosci 1:41-50.

Farinelli M, Deschaux O, Hugues S, Thevenet A, Garcia R (2006) Hippocampal train stimulation modulates recall of fear extinction independently of prefrontal cortex synaptic plasticity and lesions. Learn Mem 13:329-334.

Fleischmann A, Hvalby O, Jensen V, Strekalova T, Zacher C, Layer LE, Kvello A, Reschke M, Spanagel R, Sprengel R, Wagner EF, Gass P (2003) Impaired long-term memory and NR2A-type NMDA receptor-dependent synaptic plasticity in mice lacking c-Fos in the CNS. J Neurosci 23:9116-9122.

Floresco SB, Seamans JK, Phillips AG (1997) Selective roles for hippocampal, prefrontal cortical, and ventral striatal circuits in radial-arm maze tasks with or without a delay. J Neurosci 17:1880-1890.

Frankland PW, Bontempi B, Talton LE, Kaczmarek L, Silva AJ (2004) The involvement of the anterior cingulate cortex in remote contextual fear memory. Science 304:881-883.

Fyhn M, Molden S, Witter MP, Moser EI, Moser MB (2004) Spatial representation in the entorhinal cortex. Science 305:1258-1264.

Gisquet-Verrier P, Delatour B (2006) The role of the rat prelimbic/infralimbic cortex in working memory: not involved in the short-term maintenance but in monitoring and processing functions. Neuroscience 141:585-596

Godsil BP, Stefanacci L, Fanselow MS (2005) Bright light suppresses hyperactivity induced by excitotoxic dorsal hippocampus lesions in the rat. Behav Neurosci 119:1339-1352.

Gold AE, Kesner RP (2005) The role of the CA3 subregion of the dorsal hippocampus in spatial pattern completion in the rat. Hippocampus 15:808-814.

Guzowski JF, Setlow B, Wagner EK, McGaugh JL (2001) Experiencedependent gene expression in the rat hippocampus after spatial learning: a comparison of the immediate-early genes Arc, c-fos, and zif268. J Neurosci 21:5089-5098.

Hafting T, Fyhn M, Molden S, Moser MB, Moser EI (2005) Microstructure of a spatial map in the entorhinal cortex. Nature 436:801-806.

Hasselmo ME, Schnell E, Barkai E (1995) Dynamics of learning and recall at excitatory recurrent synapses and cholinergic modulation in rat hippocampal region CA3. J Neurosci 15:5249-5262.

Hok V, Save E, Lenck-Santini PP, Poucet B (2005) Coding for spatial goals in the prelimbic/infralimbic area of the rat frontal cortex. Proc Natl Acad Sci USA 102:4602-4607.

Jay TM, Witter MP (1991) Distribution of hippocampal CA1 and subicular efferents in the prefrontal cortex of the rat studied by means of anterograde transport of Phaseolus vulgaris-leucoagglutinin. J Comp Neurol 313:574-586

Jones MW, Wilson MA (2005) Theta rhythms coordinate hippocampalprefrontal interactions in a spatial memory task. PLoS Biol 3:e402.

Kyd RJ, Bilkey DK (2003) Prefrontal cortex lesions modify the spatial properties of hippocampal place cells. Cereb Cortex 13:444-451.

Lee I, Kesner RP (2002) Differential contribution of NMDA receptors in hippocampal subregions to spatial working memory. Nat Neurosci 5:162-168.

Lee I, Kesner RP (2003) Time-dependent relationship between the dorsal hippocampus and the prefrontal cortex in spatial memory. J Neurosci 23:1517-1523.

Lee I, Yoganarasimha D, Rao G, Knierim JJ (2004) Comparison of population coherence of place cells in hippocampal subfields CA1 and CA3. Nature 430:456-459.

Leutgeb S, Leutgeb JK, Treves A, Moser MB, Moser EI (2004) Distinct ensemble codes in hippocampal areas CA3 and CA1. Science 305:1295-1298

Leutgeb S, Leutgeb JK, Moser EI, Moser MB (2006) Fast rate coding in hippocampal CA3 cell ensembles. Hippocampus 16:765-774.

Marr D (1971) Simple memory: a theory for archicortex. Philos Trans R Soc Lond B Biol Sci 262:23-81.

Maviel T, Durkin TP, Menzaghi F, Bontempi B (2004) Sites of neocortical reorganization critical for remote spatial memory. Science 305:96-99.

Morris RG, Garrud P, Rawlins JN, O'Keefe J (1982) Place navigation impaired in rats with hippocampal lesions. Nature 297:681-683.

Moser MB, Moser EI (1998) Functional differentiation in the hippocampus. Hippocampus 8:608-619.

Nakazawa K, Quirk MC, Chitwood RA, Watanabe M, Yeckel MF, Sun LD, Kato A, Carr CA, Johnston D, Wilson MA, Tonegawa S (2002) Requirement for hippocampal CA3 NMDA receptors in associative memory recall. Science 297:211-218.

Nakazawa K, Sun LD, Quirk MC, Rondi-Reig L, Wilson MA, Tonegawa S (2003) Hippocampal CA3 NMDA receptors are crucial for memory acquisition of one-time experience. Neuron 38:305-315.

Nakazawa K, McHugh TJ, Wilson MA, Tonegawa S (2004) NMDA receptors, place cells and hippocampal spatial memory. Nat Rev Neurosci 5:361-372.

O'Keefe J, Dostrovsky J (1971) The hippocampus as a spatial map. Preliminary evidence from unit activity in the freely-moving rat. Brain Res 34:171-175.

Pace TW, Gaylord R, Topczewski F, Girotti M, Rubin B, Spencer RL (2005) Immediate-early gene induction in hippocampus and cortex as a result of novel experience is not directly related to the stressfulness of that experience. Eur J Neurosci 22:1679-1690.

Paxinos G, Watson C (1998) The rat brain in stereotaxic coordinates. San Diego: Academic

Pearce JM, Roberts AD, Good M (1998) Hippocampal lesions disrupt navigation based on cognitive maps but not heading vectors. Nature 396:75-77.

Porter MC, Burk JA, Mair RG (2000) A comparison of the effects of hippocampal or prefrontal cortical lesions on three versions of delayed nonmatching-to-sample based on positional or spatial cues. Behav Brain Res 109:69-81.

Quirk GJ, Russo GK, Barron JL, Lebron K (2000) The role of ventromedial prefrontal cortex in the recovery of extinguished fear. J Neurosci 20:6225-6231.

Ragozzino ME, Adams S, Kesner RP (1998) Differential involvement of the dorsal anterior cingulate and prelimbic-infralimbic areas of the rodent prefrontal cortex in spatial working memory. Behav Neurosci 112:293-303.

Ragozzino ME, Wilcox C, Raso M, Kesner RP (1999) Involvement of rodent prefrontal cortex subregions in strategy switching. Behav Neurosci 113:32-41.

Rowe JB, Toni I, Josephs O, Frackowiak RS, Passingham RE (2000) The 
prefrontal cortex: response selection or maintenance within working memory? Science 288:1656-1660.

Siapas AG, Lubenov EV, Wilson MA (2005) Prefrontal phase locking to hippocampal theta oscillations. Neuron 46:141-151.

Steele RJ, Morris RG (1999) Delay-dependent impairment of a matchingto-place task with chronic and intrahippocampal infusion of the NMDAantagonist D-AP5. Hippocampus 9:118-136.

Teather LA, Packard MG, Smith DE, Ellis-Behnke RG, Bazan NG (2005) Differential induction of c-Jun and Fos-like proteins in rat hippocampus and dorsal striatum after training in two water maze tasks. Neurobiol Learn Mem 84:75-84.

Teitelbaum H, Milner P (1963) Activity changes following partial hippocampal lesions in rats. J Comp Physiol Psychol 56:284-289.

Thierry AM, Gioanni Y, Degenetais E, Glowinski J (2000) Hippocampo- prefrontal cortex pathway: anatomical and electrophysiological characteristics. Hippocampus 10:411-419.

Treves A, Rolls ET (1994) Computational analysis of the role of the hippocampus in memory. Hippocampus 4:374-391.

Tsien JZ, Huerta PT, Tonegawa S (1996) The essential role of hippocampal CA1 NMDA receptor-dependent synaptic plasticity in spatial memory. Cell 87:1327-1338.

Vazdarjanova A, Guzowski JF (2004) Differences in hippocampal neuronal population responses to modifications of an environmental context: evidence for distinct, yet complementary, functions of CA3 and CA1 ensembles. J Neurosci 24:6489-6496.

Vertes RP (2006) Interactions among the medial prefrontal cortex, hippocampus and midline thalamus in emotional and cognitive processing in the rat. Neuroscience 142:1-20. 